\title{
Article \\ The Interplay between Diradical Character and Stability in Organic Molecules
}

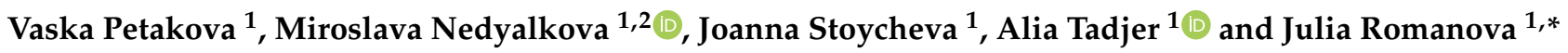 \\ 1 Faculty of Chemistry and Pharmacy, Sofia University, J. Bourchier Boulevard 1, 1164 Sofia, Bulgaria; \\ vpetakova@office365student.uni-sofia.bg (V.P.); nhmn@chem.uni-sofia.bg (M.N.); \\ jstoycheva@chem.uni-sofia.bg (J.S.); tadjer@chem.uni-sofia.bg (A.T.) \\ 2 Department of Chemistry, University of Fribourg, Chemin du Musée 9, 1700 Fribourg, Switzerland \\ * Correspondence: jromanova@chem.uni-sofia.bg
}

check for updates

Citation: Petakova, V.; Nedyalkova, M.; Stoycheva, J.; Tadjer, A.;

Romanova, J. The Interplay between Diradical Character and Stability in Organic Molecules. Symmetry 2021, 13, 1448. https://doi.org/10.3390/ sym 13081448

Academic Editor: Anthony Harriman

Received: 19 July 2021

Accepted: 3 August 2021

Published: 7 August 2021

Publisher's Note: MDPI stays neutral with regard to jurisdictional claims in published maps and institutional affiliations.

Copyright: (c) 2021 by the authors. Licensee MDPI, Basel, Switzerland. This article is an open access article distributed under the terms and conditions of the Creative Commons Attribution (CC BY) license (https:// creativecommons.org/licenses/by/ $4.0 /)$.

\begin{abstract}
The number of scientific papers on the unique properties and the potential for various applications of compounds with a diradical character is growing constantly. The diradical character enhances and even engenders certain desired optical properties and its modulation is a modern molecular design strategy. Nowadays, molecules with a non-zero diradical character are regarded as promising materials for new-generation and highly efficient solar cells and photonics devices. What is the price, however, of the unique properties of open-shell compounds? Alongside all the benefits, the diradical character is usually associated with low stability and high reactivity-unwanted molecular qualities for practical purposes. Thus, from a fundamental and applied point of view, it is important to investigate the correlation between the diradical character and laboratory stability, which is the goal of the present paper. Here, we report a combined quantum-chemical study (conceptual DFT and spin-projected HF theory) and multivariate analysis of the diradical character of a series of $o_{-}$ and $p$-quinomethides, for the stability of which experimental data are available. Our results reveal that a compromise between the diradical character and laboratory stability of a molecule is feasible and that the relationship between these two quantities can be understood in the framework of Clar's sextet theory.
\end{abstract}

Keywords: open shell; broken symmetry; conceptual DFT; chemometrics; singlet fission; photovoltaics; photonics

\section{Introduction}

The diradical character (DRC) determines the chemical reactivity and stability of molecules and produces their unique optical, magnetic, and electronic properties [1]. It varies between zero (for closed-shell systems) and one (for diradicals) and is related to the fundamental understanding of the chemical bond. The diradical character was originally defined as twice the weight of the doubly excited configuration in the singlet ground state wavefunction within the multiconfigurational self-consistent field theory and is not a directly observable feature experimentally [2]. In 2010, the connection between the DRC and spectroscopically measured quantities was demonstrated, which allowed the experimental estimation of the DRC [3]. To the best of our knowledge, however, in the literature, there are few examples of DRC evaluated by spectroscopic measurements.

Recently, it was demonstrated that the tunability of the DRC represents a fruitful computational approach for the discovery of new organic materials [4-8]. For example, molecules with a moderate (0.5) diradical character, i.e., diradicaloids, possess unusually high values of the second hyperpolarizability and are attractive for application in organic photonics [9-12]. It was also shown that compounds with a low-to-intermediate (0.1-0.5) diradical character are potential singlet fission materials [13-16]. Singlet fission is a phenomenon in which one absorbed photon is converted into two excitons. When implemented in a solar cell, it can double the number of charge carriers [17]. Therefore, 
molecules able to transform one photon into two excitons are regarded as next-generation, highly efficient organic photovoltaic materials [18-21]. From a thermodynamics point of view, in singlet fission chromophores, twice the excitation energy to the first excited triplet state, $\mathrm{E}\left(\mathrm{T}_{1}\right)$, should be less or equal to the excitation energy to the first excited singlet state, $\mathrm{E}\left(\mathrm{S}_{1}\right): 2 \mathrm{E}\left(\mathrm{T}_{1}\right)-\mathrm{E}\left(\mathrm{S}_{1}\right) \leq 0$. It was shown that the thermal losses in singlet fission are proportional to $12 \mathrm{E}\left(\mathrm{T}_{1}\right)-\mathrm{E}\left(\mathrm{S}_{1}\right) 1$ and, hence, zero or slightly smaller differences are required from a practical point of view [4]. Since the first triplet excitation energy is also inversely proportional to the DRC, it is not surprising that molecules with a DRC between 0.1 and 0.5 provide optimal $2 \mathrm{E}\left(\mathrm{T}_{1}\right)-\mathrm{E}\left(\mathrm{S}_{1}\right)$ values for real photovoltaics application.

Alongside attractive properties, the diradical character is usually an implication for high molecular reactivity and instability [22]. Here we arrive at an intrinsic paradox: the presence of the diradical character, which makes a substance prospective for photovoltaics and non-linear optics, may also disqualify the material as inappropriate for application. A deeper analysis of this contradiction reveals that it expresses the balance between the properties of the ground and the excited states.

Molecular stability is usually understood in two aspects: thermodynamic and kinetic. The thermodynamic stability is determined solely by the molecular structure and is related to the Gibbs energy, which is governed by the total electron energy of the molecule [23]. Thus, the diradical character depends on the HOMO-LUMO gap (where HOMO is the highest occupied molecular orbital and LUMO is the lowest unoccupied molecular orbital) and, as an electronic characteristic, is associated with the thermodynamic stability of the compound. The kinetic stability is also structure-dependent and is defined by the chemical reactivity of the molecules and the rates of the reactions in which they participate [23]. The chemical reactivity depends on the ionization potential, the electron affinity, the charge and spin distribution (electron density) in a molecule and, hence, is correlated with its diradical character as well. A kinetically unstable (highly reactive) molecule may have a fairly long lifetime if kept away from agents for which it has affinity (e.g., in a vacuum). A thermodynamically unstable molecule, however, even if completely isolated, has a short lifetime because, to stabilize, it will undergo structural changes (e.g., Jahn-Teller effect) or it may never become synthetically feasible.

To date, the relationship between reactivity, stability, and the diradical character remains an intriguing topic from a fundamental perspective [22]. The present theoretical study aims to contribute to the deeper understanding of the problem and to explore whether there are stability limitations to the application of molecules with a non-zero DRC. For this purpose, we combine quantum-chemical and chemometrics approaches to treat a family of quinone methides, which differ in laboratory stability and reactivity. To the best of our knowledge, this is the first theoretical study to focus on the intimate relationship between the diradical character and laboratory stability of molecules.

\section{Materials and Methods}

\subsection{Molecular Set}

The study is based on an old, but fruitful for the purpose, paper of Musil et al. who tried to explain the laboratory stability of quinone methides applying Hückel theory, i.e., with respect to aromaticity [24]. A valuable contribution of the authors is the summary of the literature data on the laboratory stability of a large number of structurally similar but markedly differing in reactivity conjugated compounds (Figure 1). Presented are also the mechanisms of the reactions in which the quinone methides participate, causing their laboratory instability. The occurrence of an exocyclic methide group most often initiates reactions with nucleophiles (water) or with a radical mechanism (dimerization). The authors define as stable those quinone methides that can be isolated and have infinite lifetime, allowing detailed examination of their properties. Moderate stability applies for quinone methides that can exist only in solution or have a limited lifetime (minutes or hours) but long enough to allow spectroscopic validation and investigation. As unstable are classified the highly reactive quinone methides with too short lifetimes, which makes 
their experimental characterization impossible. Some analogous compounds differ only in the type of substituents and therefore have two versions labelled as ' $a$ ' for the hydrogen substituent and ' $b$ ' for the phenyl-group substituent. Those that have no analogues bear no label and have only hydrogen substituents in the exocyclic methide group.<smiles>CC(O)=C1C=CC(=O)C=C1</smiles><smiles>O=C1C=CC=CC1=C(Br)Br</smiles><smiles>O=C1C=Cc2ccccc2C1=C(P)c1ccccc1</smiles><smiles>O=C1C=Cc2ccccc2C1=O</smiles><smiles>CC(P)=c1ccc2c(c1)C=CC(=O)C=2</smiles>

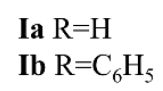

IIa $\mathrm{R}=\mathrm{H}$

IIIa $\mathrm{R}=\mathrm{H}$

IVa $\mathrm{R}=\mathrm{H}$

Va $\mathrm{R}=\mathrm{H}$

Ib $\mathrm{R}=\mathrm{C}_{6} \mathrm{H}_{5}$

IIb $\mathrm{R}=\mathrm{C}_{6} \mathrm{H}_{5}$

IIIb $\mathrm{R}=\mathrm{C}_{6} \mathrm{H}_{5}$

IVb $\mathrm{R}=\mathrm{C}_{6} \mathrm{H}_{5}$ Vb $\mathrm{R}=\mathrm{C}_{6} \mathrm{H}_{5}$<smiles>CC(C)=C1C=c2ccccc2=CC1=O</smiles>

VIa $\mathrm{R}=\mathrm{H}$ VIb $\mathrm{R}=\mathrm{C}_{6} \mathrm{H}_{5}$<smiles>O=C1C=CC(=C(P)P)c2ccccc21</smiles>

VIIa $\mathrm{R}=\mathrm{H}$ VIIb $\mathrm{R}=\mathrm{C}_{6} \mathrm{H}_{5}$<smiles>C=c1cccc2c1=CC=CC2=O</smiles>

VIII<smiles>C=c1cccc2c1=CC(=O)C=C2</smiles>

IX<smiles>NC=c1ccc2c(c1)C(=O)C=CC=2</smiles>

X<smiles>[2H]C(P)=C1c2ccccc2C(=O)c2ccccc21</smiles>

XIa $\mathrm{R}=\mathrm{H}$ XIb $\mathrm{R}=\mathrm{C}_{6} \mathrm{H}_{5}$<smiles></smiles>

XII

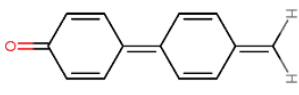

XIII

Figure 1. Quinone methides considered in this study and in the paper of Musil et al. Reproduced from [24].

\subsection{Calculation of the Diradical Character}

There are two general approaches in the theoretical prediction of DRC: the use of multiconfigurational methods such as RASSCF/CASSCF [25] or the application of the spin-projected unrestricted Hartree-Fock (PUHF) method [2] with natural orbital analysis [26]. The multiconfigurational methods are highly accurate for open-shell systems and they can correctly predict the DRC of a singlet fission chromophore, as well as all properties that determine the application potential of a molecule. However, the multiconfigurational methods are heavily resource-demanding and usually limited to small or medium molecules. The PUHF method allows finding the broken symmetry singlet solution and, in combination with natural orbital analysis, can be used for DRC calculations even for very large molecules at a negligible computational cost. PUHF is an approximated method for the treatment of open-shell systems and, while it accurately predicts the DRC, it cannot estimate a variety of other molecular properties. In the framework of the PUHF theory, the diradical character $\left(\mathrm{y}_{0}, \mathrm{i}=0\right)$ depends on the occupation numbers of HONO (highest occupied natural orbital) and LUNO (lowest unoccupied natural orbital):

$$
y_{i}=1-\frac{2 T_{i}}{1+T_{i}^{2}}
$$

where $T_{i}$ is the overlap integral of the respective orbital pair, which is calculated using the occupation numbers $(n)$ of the natural orbitals of the UHF wavefunction:

$$
\boldsymbol{T}_{i}=\frac{\boldsymbol{n}_{\mathrm{HONO}-i}-\boldsymbol{n}_{\mathrm{LUNO}+i}}{2}
$$




\subsection{Quantum-Chemical Protocol}

Our computational protocol encompasses geometry optimization of the molecules with B3LYP $/ 6-31 G^{*}$, followed by frequency analysis [27-30]. Next, the optimized structures are subject to single point "broken symmetry" calculations with the PUHF/6-31G method in combination with natural orbital population analysis, from which the diradical character is quantified. All calculations were performed with Gaussian 09 [31]. The chemical reactivity, the global and local reactivity indices are calculated within the conceptual density functional theory (CDFT) [32]. CDFT is a popular and well-proven approach for the extraction of chemically relevant principles from DFT, in particular for interpretation of the chemical reactivity of the molecules. All CDFT data reported in the manuscript were obtained using the program Multiwfn 3.7 [33]. In order to calculate the CDFT quantities for a given molecule, the Multiwfn 3.7 program requires as input three separate wavefunctions with molecular charges of $0,+1$, and -1 . These wavefunctions were obtained from B3LYP $/ 6-31 \mathrm{G}^{*}$ single-point calculations on the optimized geometry with the Gaussian 09 software. The computed values of the local and global reactivity indices and their definitions are collected in Table 1. For more details on the approximations used for the calculation of all CDFT quantities, the readers are referred to the manual of the Multiwfn 3.7 program and all related references therein.

Table 1. Global and local reactivity indices computed within the CDFT framework [33]. $\mathrm{N}$ is the number of electrons in a molecule.

\begin{tabular}{|c|c|c|c|}
\hline \multicolumn{2}{|l|}{ Global (B3LYP/6-31G*) } & \multicolumn{2}{|c|}{ Local (B3LYP/6-31G*) with Hirschfeld Charges } \\
\hline $\mathbf{I}=\mathrm{E}(\mathrm{N}-1)-\mathrm{E}(\mathrm{N})$ & Vertical ionization potential & $\mathbf{f}^{+} \max$ & Largest atomic Fukui index for nucleophilic attack \\
\hline$A=E(N)-E(N+1)$ & Vertical electron affinity & $\mathbf{f}^{-} \max$ & Largest atomic Fukui index for electrophilic attack \\
\hline$\eta=\mathrm{E}_{\mathrm{LUMO}}-\mathrm{E}_{\mathrm{HOMO}}$ & Hardness & $\mathbf{f}_{\max }$ & Largest atomic Fukui index for radical attack \\
\hline$\delta=\frac{1}{\eta}$ & Softness & & \\
\hline$\chi=-\frac{1}{2}\left(\mathrm{E}_{\mathrm{HOMO}}+\mathrm{E}_{\mathrm{LUMO}}\right)$ & Electronegativity & & \\
\hline $\boldsymbol{\omega}=\frac{\chi 2}{2 \eta}$ & Electrophilicity & & \\
\hline
\end{tabular}

In addition to the computed global and local reactivity indices, we adopted all quantities obtained by Musil et al. with Hückel theory, namely, REPE (resonance energy per $\pi$-electron), $\Delta E_{i}, \Delta E_{i j}$ (reactivity indices), and $P$ (stability index) [24]. The stability index is a combined quantity that we derived to explain the laboratory stability of quinone methides and is calculated as follows:

$$
\boldsymbol{P}=437,4 \frac{\boldsymbol{R E P E}}{\Delta \boldsymbol{E}_{i} \Delta \boldsymbol{E}_{i j}}
$$

Based on the stability index, the authors [24] have succeeded to predict and synthesize moderately stable (IIb, $\mathbf{V b}$ and $\mathbf{V I b}$ ) and unstable (VIa) quinone methides.

Complementing Hückel theory, another iconic rule is used for interpretation of the results, namely, Clar's rule, stating that: the Kekule resonance structure with the largest number of disjoint aromatic $\pi$-sextets is the most important for characterization of the properties of polycyclic compounds; the aromatic $\pi$-sextets are defined as six $\pi$-electrons localized in a single benzene-like ring separated from adjacent rings by formal C-C single bonds [34].

\subsection{Multivariate Analysis}

The combination of multivariate statistical methods with atomistic simulation and modeling has become a useful tool in the field of structure-property classification and prediction models [35-37]. The classification model used in this study is based on the grid of property descriptors described in the previous section. The hierarchical cluster analysis (HCA) and a non-hierarchical (K-means) method were used as exploratory data analysis methods to reveal the main separation and discriminating factors for the dataset. 
As an unsupervised clustering method, HCA is employed to gain a better insight into data structures; the formed groups (clusters) are based on a similarity measure (i.e., Euclidean distance) without considering the class-membership function $[38,39]$. The graphical representation of the clustering results can be displayed in a tree-like structure, the dendrogram, which can be used to probe and recognize the similarity patterns and clusters within objects and variables in a feature space.

To conduct unsupervised learning, non-hierarchical clustering is applied. K-means clustering is one of the common unsupervised machine learning algorithms, applied for a plethora of problems. The K-means algorithm classifies $\mathrm{n}$ observations into $\mathrm{k}$ clusters grouped around centroids by assigning every data point from the input matrix to the nearest cluster, keeping the distance to the centroids as short as possible. The number $\mathrm{k}$ denotes the centroids, and the centroid is the real or imaginary location indicating the center of the cluster. Combined sequentially, the two approaches can efficiently use the local descriptor environment generated by quantum chemistry calculation and can produce a satisfying and chemically accurate classification model revealing patterns of similarity between the objects of study. All chemometric calculations were performed with the software package STATISTICA 8.0 [40].

\section{Results and Discussion}

The results of our study on quinone methides are presented in Figure 2, while Figure 3 summarizes the results reported by Musil et al. [24]. All numerical data are presented in the Supplementary Materials (SM), Table S1.

Figure 2 clearly illustrates that all the quinone methides considered have a non-zero diradical character. DRC varies in the series in the range 0.07-0.51 and, consequently, with respect to the requirements for singlet fission chromophores, these compounds are potential photovoltaics. The results for all stable quinone methides isolated in the crystal phase are coloured in green and they feature DRC values in the interval of $0.07-0.20$. For comparison, the respective values for the unstable species vary within the range of 0.11-0.40. Consequently, the laboratory stability of quinone methides cannot be explained solely with the DRC values. Particularly illustrative is compound VIa, featuring one of the highest DRC values in the series, notwithstanding exhibiting moderate laboratory stability. Another interesting observation is that the functionalization of the exocyclic carbon with two phenyl substituents results, in most cases, in augmented DRC (by 0.08 on average) concurrent with enhanced kinetic stability.

Among the global reactivity indices, the global hardness mirrors the DRC values, while the softness changes synchronously with the DRC. Considering the definitions of hardness and softness (Table 1), this is an expected result: molecules with high softness/low hardness have a narrow HOMO-LUMO gap, hence, a pronounced diradical character.

It is important to point out the parallel between the diradical character and the electron affinity. The compounds with strong electron affinity, i.e., with low LUMOs, are those with a large DRC. In the language of chemistry, strong electron affinity signifies an easy reduction. This relationship is not unexpected, since, in some alternative approaches for DRC assessment, the DRC is defined as the occupation number of LUNO, which increases with the decrease in LUNO energy. Correspondingly, the low ionization potential indicates a high HOMO, and this, combined with a low LUMO, results in a narrow HOMO-LUMO gap and a substantial DRC. In most cases, the low ionization potential is related to a large DRC, but the ionization potential alone cannot explain the variations in the DRC. The electronegativity essentially follows the profile of the ionization potential, while the electrophilicity matches softness. 

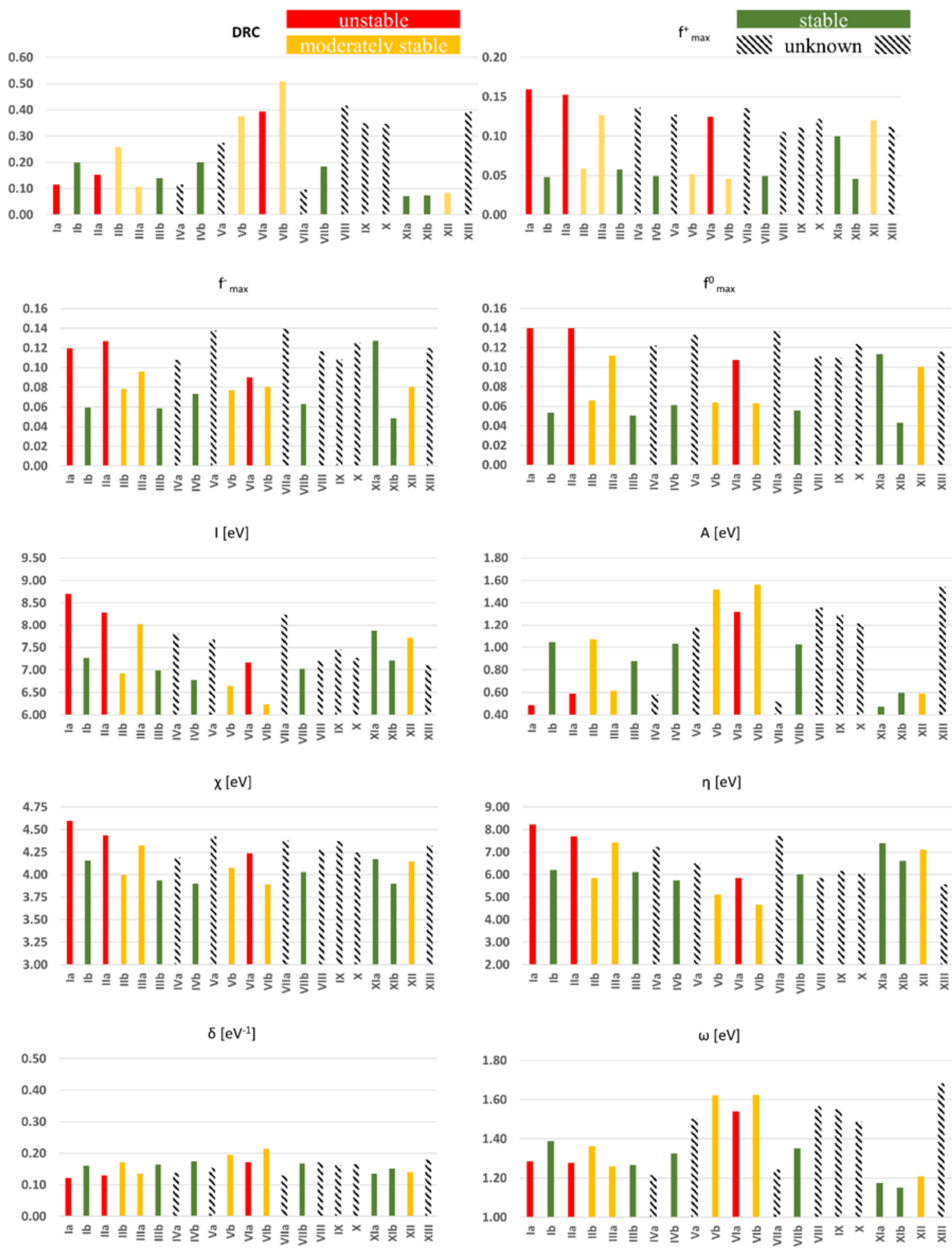

Figure 2. Diradical character (DRC) calculated with PUHF/6-31G**. Global and local reactivity indices (Table 1) calculated in this study in the framework of CDFT with the B3LYP/6-31G* method: I [eV]—vertical ionization potential, A [eV]—vertical electron affinity, $\chi[\mathrm{eV}]$ —electronegativity, $\eta[\mathrm{eV}]$ —hardness, $\delta\left[\mathrm{eV}^{-1}\right]$ —softness, $\omega[\mathrm{eV}]$ —electrophilicity, $\mathrm{f}^{+}$max - largest atomic Fukui index for electrophilic attack, $\mathrm{f}^{-}$max - largest atomic Fukui index for nucleophilic attack, and $\mathrm{f}^{0}{ }_{\max }-$ largest atomic Fukui index for radical attack. 


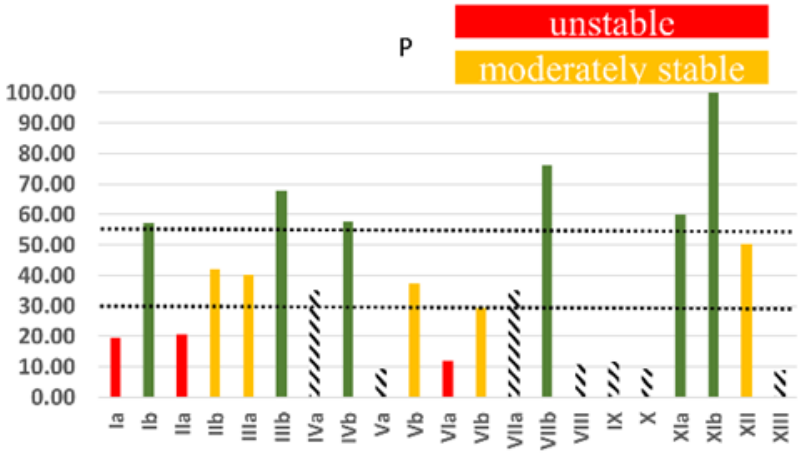

$\Delta \mathrm{E}_{\mathrm{i}}\left[\beta^{-1}\right]$

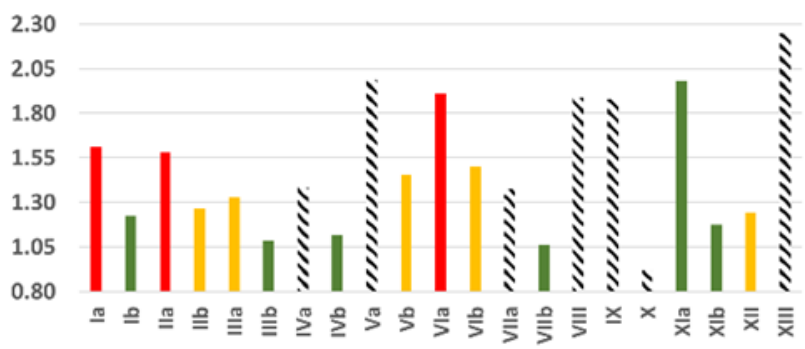

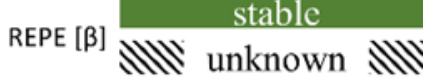

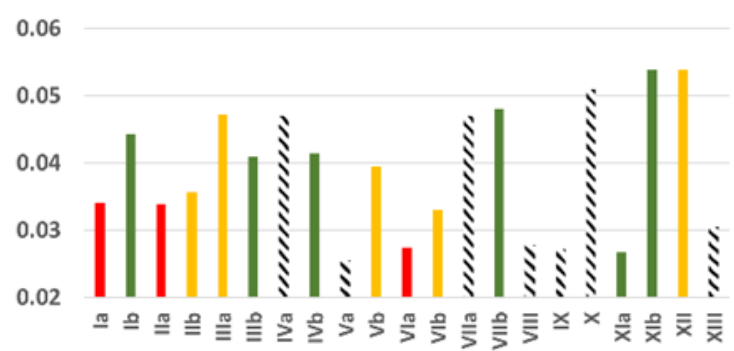

$\Delta \mathrm{E}_{\mathrm{ij}}\left[\beta^{-1}\right]$

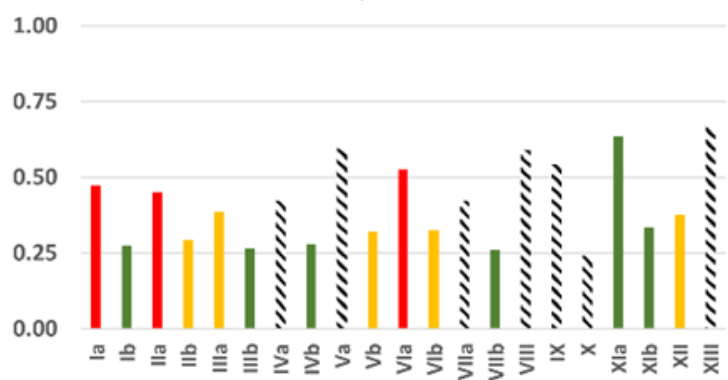

Figure 3. Theoretical quantities calculated by Musil et al. utilizing the Hückel method for quinone methides [24]: $\mathrm{P}$-generalized stability index, REPE $[\beta]$-resonance energy per $\pi$ electron, and $\Delta E_{i}\left[\beta^{-1}\right], \Delta E_{i j}\left[\beta^{-1}\right]$ 一reactivity indices. The experimentally observed laboratory stability is represented by the color code. Highly stable quinone methides have $\mathrm{P}>55$ and they were obtained experimentally in the crystal phase. Compounds with $30<\mathrm{P}<55$ have moderate stability, and those with $\mathrm{P}<30$ are unstable.

For clarification of the laboratory stability of quinone methides, analysis of the local reactivity indices was needed in addition to the DRC. Taking into account the knowledge about the characteristic reaction mechanisms for this class of compounds, most important should be the Fukui indices for nucleophilic (water) and radical (dimerization) attacks. As anticipated, in the compounds without substituents in the exocyclic methylene group (the $a$ series), the carbon atom in the latter group is the most reactive. In general, the $a$ series features higher values of the local Fukui indices than the substituted analogs ( $\boldsymbol{b}$ series). The comparison of DRC with $\mathrm{f}^{+}$max outlines the following extreme cases:

(1) low DRC (0.114) + high $\mathrm{f}^{+}$max $(0.160)=$ instability (Ia)

(2) low DRC (0.084) + medium-to-high $\mathrm{f}^{+}{ }_{\max }(0.120)=$ moderate stability (XII)

(3) low DRC (0.074) + low $\mathrm{f}^{+}$max $(0.046)=$ stability $(\mathbf{X I b})$

(4) medium DRC (0.394) + medium-to-high $\mathrm{f}^{+}{ }_{\max }(0.125)=$ instability (VIa)

(5) medium DRC (0.508) + low $\mathrm{f}^{+}{ }_{\max }(0.046)=$ moderate stability (VIb).

As the Fukui indices for the three types of attack display analogous behavior within each series, the same relations apply for all three.

The analysis reveals a fine balance between the DRC and reactivity, which may result in both stable and unstable compounds with an open-shell character. To further examine the complex interrelations within each series, we performed a chemometrics itemization of the data employing cluster analysis. A table with all descriptors and compounds subject to the cluster analysis is presented in the SM, Table S1.

The chemometrics analysis results are presented in Figure 4 (as well as in the SM, Figures S1-S2, Tables S2-S4) and show a very good separation of the quinone methides based on their electronic properties, reactivity, and stability. Three groups emerge: Cluster 1, Cluster 2, and Cluster 3. Cluster 3 encompasses compounds Ia, IIa, IIIa, IVa, VIIa, Xia, and XII. This is the group of the low-to-moderate stable molecules. All compounds in this cluster belong to the $\boldsymbol{a}$ series and contain a highly reactive non-substituted exocyclic carbon. 
They feature lowest electron affinity, highest ionization potential (prominent reducers), and, hence, lowest softness and electrophilicity. Their averaged diradical character amounts to 0.11 and is the lowest for the series range. However, they are also characterized by the largest atomic Fukui indices and are therefore highly reactive. The compounds from Cluster 3 are not interesting for application because they do not possess a significant diradical character or laboratory stability.

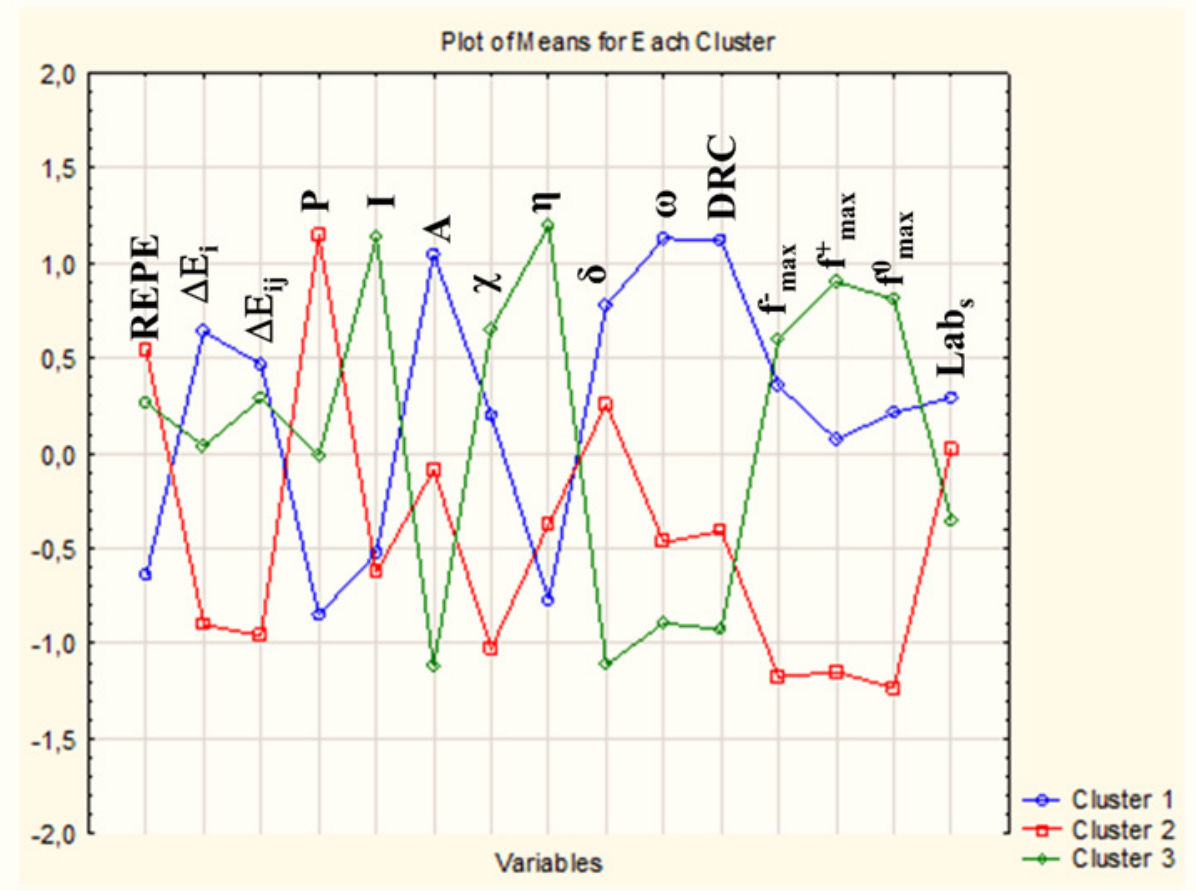

Figure 4. K-means clustering of quinone methides (the dataset is in the SM, Table S1): REPE [ $\beta]$-resonance energy per $\pi$ electron, $\Delta E_{i}\left[\beta^{-1}\right]$ and $\Delta E_{i j}\left[\beta^{-1}\right]$-Hückel reactivity indices, $\mathrm{P}-$ generalized stability index, $\mathrm{I}[\mathrm{eV}]$ —vertical ionization potential, $\mathrm{A}[\mathrm{eV}]$ —vertical electron affinity, $\chi$ $[\mathrm{eV}]$ —electronegativity, $\eta[\mathrm{eV}]$ —-hardness, $\delta\left[\mathrm{eV}^{-1}\right]$ —softness, $\omega[\mathrm{eV}]$ —electrophilicity, $\mathrm{f}^{-}$max —largest atomic Fukui index for electrophilic attack, $\mathrm{f}^{+}$max largest atomic Fukui index for nucleophilic attack, $\mathrm{f}^{0}$ max -largest atomic Fukui index for radical attack, and Lab $\mathrm{s}_{\mathrm{s}}$-laboratory stability.

Cluster 2 contains the moderate-to-high stability compounds and comprises all molecules from the $\boldsymbol{b}$ series: $\mathbf{I} \mathbf{b}, \mathbf{I I} \mathbf{b}, \mathbf{I I I} \mathbf{b}, \mathbf{I V b}, \mathbf{V I I} \mathbf{b}$, and $\mathbf{X I} \mathbf{I b}$. All these quinone methides have structural analogues from Cluster 3 . The average diradical character of the compounds in Cluster 2 is 0.18 (i.e., higher than that for Cluster 3), but their lower reactivity, and hence higher stability, are ensured by phenyl functionalization of the methylene carbon. The latter is obvious from the lowest values of the atomic Fukui indices, too. The molecules from Cluster 2 are also characterized by the lowest ionization potential and moderate electron affinity, which results in moderate-to-high softness in the series. Cluster 2 is a good example of a reasonable compromise between a relatively significant diradical character and stability. This result clearly demonstrates that stable molecular materials with DRC exist, and that the combination between quantum-chemical and chemometrics approaches is a powerful tactic to find them. Finally, compounds Va, Vb, VIb, VIa, VIb, VIII, IX, $\mathbf{X}$, and XIII belong to Cluster 1, which differentiates the group of the moderately-stabileto-unstable molecules. In the whole series, these compounds have the highest electron affinities (prominent oxidizers), lowest hardness, highest softness and electrophilicity. Their averaged DRC is intermediate, 0.38 , which is also a high value for the series. Therefore, although attractive from a properties perspective, such compounds are useless in practice.

From the discussion on the quantum-chemical results, it is clear that the used descriptors are correlated. This correlation, however, does not have a negative effect on the clustering (hierarchical or K-means) since cluster labels are assigned on the basis of the 
Euclidean distance between the points in the clusters and their corresponding centroids in the 15-dimensional feature space.

Inspection of the ground state resonance structures discloses that the distinct partition of quinone methides in terms of electronic properties and laboratory stability can be interpreted by implementing Clar's rule [34,41] for the aromatic sextets (Figure 5).

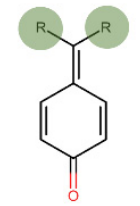

Ia $\mathrm{R}=\mathrm{H}$ Ib $\mathrm{R}=\mathrm{C}_{6} \mathrm{H}_{5}$

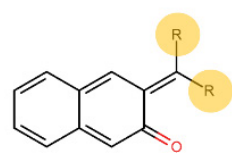

VIa $\mathrm{R}=\mathrm{H}$ VIb $\mathrm{R}=\mathrm{C}_{6} \mathrm{H}_{5}$

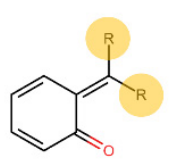

IIa $\mathrm{R}=\mathrm{H}$

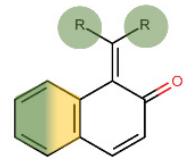

IIIIa $\mathrm{R}=\mathrm{H}$

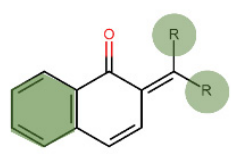

IVa $\mathrm{R}=\mathrm{H}$

IVb $\mathrm{R}=\mathrm{C}_{6} \mathrm{H}_{5}$<smiles>CC(Br)=c1ccc2c(c1)C=CC(=O)C=2</smiles>

Va $\mathrm{R}=\mathrm{H}$

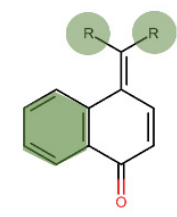

VIIa $\mathrm{R}=\mathrm{H}$

VIIIb $\mathrm{R}=\mathrm{C}_{6} \mathrm{H}_{5}$<smiles>C=c1cccc2c1=CC=CC2=O</smiles>

VIII<smiles>C=c1cccc2c1=CC(=O)C=C2</smiles>

IX<smiles>O=C1C=CC=c2ccc(=CO)cc21</smiles>

$\mathbf{X}$

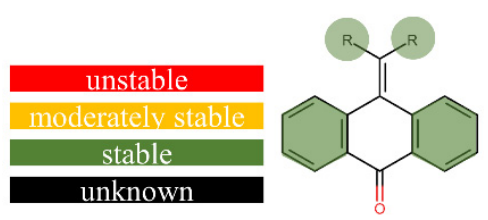

XIa $\mathrm{R}=\mathrm{H}$

XIb $\mathrm{R}=\mathrm{C}_{6} \mathrm{H}_{5}$<smiles>CC=C1C(=O)c2ccccc2-c2ccccc21</smiles>

XII

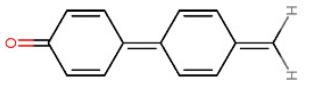

XIII

Figure 5. Kekulé structures of quinone methides containing Clar sextets (shaded) having a stabilizing effect on the ground state. The experimentally observed laboratory stability of the ground state is represented a color code and the Clar sextets are shaded correspondingly. All bi- and tricyclic compounds without Clar sextets in the basic structure belong to Cluster 1, the group of the unstable molecules. The rest have at least one Clar sextet in the quinone methide fragment and fall in Cluster 2 and 3-the groups of stable and moderately stable molecules.

All stable and moderately stable compounds (Cluster 2 and 3) possess at least one Clar sextet in the main quinone methide structure (excluding the phenyl substituents). $\mathbf{I b}$ and IIb are an exception, but in these, the stabilization originates from the two phenyl substituents that import two Clar sextets, thus greatly stabilizing the single six-membered quinoid ring of the main structure. For the remaining compounds, the introduction of two phenyl substituents results in no more than moderate stability. Our recent study on singlet fission chromophores also demonstrates the importance of Clar's sextet rule for the design of photovoltaics with diradical character [16]. Therefore, it is worth investing in the development of high-throughput approaches for counting Clar's sextets and their implementation in qualitative structure-property relationships models for finding new solar cell materials. To the best of our knowledge, currently, computational approaches for counting Clar's sextets exist but are limited to polycyclic aromatic hydrocarbons [42].

\section{Conclusions}

The laboratory stability of quinone methides was rationalized using their diradical character, global and local reactivity indices, chemometrics analysis, and Clar's rule. All investigated compounds possess non-zero DRCs, and the stable or moderately stable among them possess at least one Clar sextet in the resonance structure. It was established that the average DRC value of 0.18 is in accordance with laboratory stability, which demonstrates that a compromise between a non-zero diradical character and real application 
can be found. For this purpose, the theoretical investigations must include global and local reactivity indices as this class of descriptors is related to the DRC. The study reveals that the reactivity indices and Clar's sextet rule open new horizons for high-throughput materials design. They can be implemented in chemometrics approaches for the quest of stable materials for applications in singlet fission photovoltaics and photonics.

Supplementary Materials: The following are available online at https: / www.mdpi.com/article / 10.3390/sym13081448/s1, Table S1: Dataset used for the K-means clustering of quinone methides; Tables S2-S4: Members distribution in the obtained clusters from K-means clustering; Figure S1: Hierarchical clustering for the variable-dendrogram representation; Figure S2: Hierarchical clustering of the objects-a dendrogram representation.

Author Contributions: Conceptualization, J.R.; methodology, J.R. and M.N.; formal analysis, J.R., A.T., J.S. and M.N.; investigation, V.P.; writing—original draft preparation, J.R.; writing-review and editing, J.R., A.T. and J.S.; visualization, J.R. and M.N.; supervision, J.R.; project administration, J.R.; funding acquisition, J.R. All authors have read and agreed to the published version of the manuscript.

Funding: The authors acknowledge the financial support of the Bulgarian National Science Fund, contract КП-06-H39/2 from 9 December 2019; https://ml4sf.chem.uni-sofia.bg/ (accessed on 21 October 2020).

Acknowledgments: J.S. acknowledges the support, received from the "Young scientists and Postdoctoral candidates" program and the National Research Program E+: Low Carbon Energy for the Transport and Households of the Bulgarian Ministry of Education and Science, MCD No. 577/ 17 August 2018.

Conflicts of Interest: The authors declare no conflict of interest. The funders had no role in the design of the study; in the collection, analyses, or interpretation of data; in the writing of the manuscript, or in the decision to publish the results.

\section{References}

1. Nakano, M. Electronic structure of open-shell singlet molecules: Diradical character viewpoint. Top. Curr. Chem. 2017, 375, 47. [CrossRef] [PubMed]

2. Yamaguchi, K. Self-Consistent Field: Theory and Applications; Carbo, R., Klobukowski, M., Eds.; Elsevier: Amsterdam, The Netherlands, 1990; p. 727.

3. Kamada, K.; Ohta, K.; Shimizu, A.; Kubo, T.; Kishi, R.; Takahashi, H.; Botek, E.; Champagne, B.; Nakano, M. Singlet diradical character from experiment. J. Phys. Chem. Lett. 2010, 1, 937-940. [CrossRef]

4. Minami, T.; Nakano, M. Diradical character view of singlet fission. J. Phys. Chem. Lett. 2012, 3, 145-150. [CrossRef]

5. Minami, T.; Ito, S.; Nakano, M. Fundamental of diradical-character-based molecular design for singlet fission. J. Phys. Chem. Lett. 2013, 4, 2133-2137. [CrossRef]

6. Nakano, M.; Champagne, B. Theoretical design of open-shell singlet molecular systems for nonlinear optics. J. Phys. Chem. Lett. 2015, 6, 3236-3256. [CrossRef]

7. Nakano, M.; Champagne, B. Nonlinear optical properties in open-shell molecular systems. WIREs Comput. Mol. Sci. 2016, 6, 198-210. [CrossRef]

8. Nakano, M. Excitation Energies and Properties of Open-Shell Singlet Molecules: Application to a New Class of Molecules for Nonlinear Optics and Singlet Fission; Springer: Cham, Switzerland, 2014.

9. Takamuku, S.; Nakano, M. Theoretical study on second hyperpolarizabilites of intramolecular pancake-bonded diradicaloids with helical scaffolds. ACS Omega 2019, 4, 2741-2749. [CrossRef]

10. Nakano, M.; Minami, T.; Yoneda, K.; Muhammad, S.; Kishi, R.; Shigeta, Y.; Kubo, T.; Rougier, L.; Champagne, B.; Kamada, K.; et al. Giant enhancement of the second hyperpolarizabilites of open-shell singlet polyaromatic diphenalenyl diradicaloids by an external electric field and donor-acceptor substitution. J. Phys. Chem. Lett. 2011, 2, 1094-1098. [CrossRef]

11. Nakano, M.; Champagne, B. Diradical character dependences of the first and second hyperpolarizabilites of asymmetric open-shell singlet systems. J. Chem. Phys. 2013, 138, 244306. [PubMed]

12. Matsui, H.; Yamane, M.; Tonami, T.; Nakano, M.; de Wergifosse, M.; Seidler, T.; Champagne, B. Theoretical study on third-order nonlinear optical property of one-dimensional cyclic thiazyl aggregates: Intermolecular distance, open-shell nature, and spin-state dependences. J. Phys. Chem. C 2018, 122, 6779-6785. [CrossRef]

13. Stoycheva, J.; Romanova, J.; Tadjer, A. Women in singlet fission world: Pearls in a semi-open shell. Molecules 2021, $26,2922$. [CrossRef]

14. Ito, S.; Minami, T.; Nakano, M. Diradical character based design for singlet fission of condensed-ring systems with $4 \mathrm{n} \pi$ electrons. J. Phys. Chem. C 2012, 116, 19729-19736. [CrossRef] 
15. Ito, S.; Nakano, M. Theoretical molecular design of heteroacenes for singlet fission: Tuning the diradical character by modifying $\pi$-conjugation length and aromaticity. J. Phys. Chem. C 2015, 119, 148-157. [CrossRef]

16. Stoycheva, J.; Tadjer, A.; Garavelli, M.; Spassova, M.; Nenov, A.; Romanova, J. Boron-doped polycyclic aromatic hydrocarbons: A molecular set revealing the interplay between topology and singlet fission propensity. J. Phys. Chem. Lett. 2020, 11, 1390-1396. [CrossRef]

17. Smith, M.B.; Michl, J. Singlet fission. Chem. Rev. 2010, 110, 6891-6936. [CrossRef] [PubMed]

18. Zaykov, A.; Felkel, P.; Buchanan, E.A.; Jovanovic, M.; Havenith, R.W.A.; Kathir, R.K.; Broer, R.; Havlas, Z.; Michl, J. Singlet fission rate: Optimized packing of a molecular pair. Ethylene as a model. J. Am. Chem. Soc. 2019, 141, 17729-17743. [CrossRef]

19. Ryerson, J.L.; Zaykov, A.; Suarez, L.E.A.; Havenith, R.W.A.; Stepp, B.R.; Dron, P.I.; Kaleta, J.; Akdag, A.; Teat, S.J.; Magnera, T.F.; et al. Structure and photophysics of indigoids for singlet fission: Cibalackrot. J. Chem. Phys. 2019, 151, 184903. [CrossRef] [PubMed]

20. Karlsson, J.K.G.; Atahan, A.; Harriman, A.; Tojo, S.; Fujitsuka, M.; Majima, T. Pulse radiolysis of TIPS-pentacene and a fluorenebridged bis(pentacene): Evidence for intramolecular singlet-exciton fission. J. Phys. Chem. Lett. 2018, 9, 3934-3938. [CrossRef]

21. Karlsson, J.K.G.; Atahan, A.; Harriman, A.; Tkachenko, N.V.; Ward, A.D.; Schaberle, F.A.; Serpa, C.; Arnaut, L.G. Singlet exciton fission and associated enthalpy changes with a covalently linked bichromophore comprising TIPS-pentacenes held in an open conformation. J. Phys. Chem. A 2021, 125, 1184-1197. [CrossRef]

22. Stuyver, T.; Chen, B.; Zeng, T.; Geerlings, P.; de Proft, F.; Hoffmann, R. Do diradicals behave like diradicals? Chem. Rev. 2019, 119, 11291-11351. [CrossRef] [PubMed]

23. IUPAC. Compendium of Chemical Terminology, 2nd ed.; (The “Gold Book”); McNaught, A.D., Wilkinson, A., Eds.; Blackwell Scientific Publications: Oxford, UK, 1997.

24. Musil, L.; Koutek, B.; Pišová, M.; Souček, M. Delocalization and stability of o- and p-quinone methides: A HMO study. Collect. Czech. Commun. 1981, 46, 1148-1159. [CrossRef]

25. Malmqvist, P.A.; Rendell, A.; Roos, B.O. The restricted active space self-consistent-field method, implemented with a split graph unitary group approach. J. Phys. Chem. 1990, 94, 5477-5482. [CrossRef]

26. Weinhold, F.; Landis, C.R. Discovering Chemistry With Natural Bond Orbitals; John Wiley \& Sons: Hoboken, NJ, USA, 2012.

27. Becke, A.D. Density-functional thermochemistry. III. The role of exact exchange. J. Chem. Phys. 1993, 98, 5648-5652.

28. Lee, C.; Yang, W.; Parr, R.G. Development of the Colle-Salvetti correlation-energy formula into a functional of the electron density. Phys. Rev. B 1998, 37, 785-789. [CrossRef]

29. Vosko, S.H.; Wilk, L.; Nusair, M. Accurate spin-dependent electron liquid correlation energies for local spin density calculations: A critical analysis. Can. J. Phys. 1980, 58, 1200-1211. [CrossRef]

30. Stephens, P.J.; Devlin, F.J.; Chabalowski, C.F.; Frisch, M.J. Ab initio calculation of vibrational absorption and circular dichroism spectra using DFT force fields. J. Phys. Chem. 1994, 98, 11623-11627. [CrossRef]

31. Frisch, M.J.; Trucks, G.W.; Schlegel, H.B.; Scuseria, G.E.; Robb, M.A.; Cheeseman, J.R.; Scalmani, G.; Barone, V.; Mennucci, B.; Petersson, G.A.; et al. Gaussian 09, Revision E.01; Gaussian, Inc.: Wallingford, CT, USA, 2009.

32. Geerlings, P.; de Proft, F.; Langenaeker, W. Conceptual density functional theory. Chem. Rev. 2003, 103, 1793-1874. [CrossRef] [PubMed]

33. Lu, T.; Chen, F. Multiwfn: A multifunctional wavefunction analyzer. J. Comput. Chem. 2012, 33, 580-592. Available online: http:/ / sobereva.com/multiwfn/ (accessed on 5 December 2019). version 3.7. [CrossRef] [PubMed]

34. Clar, E. The Aromatic Sextet; John Wiley \& Sons: New York, NY, USA, 1972.

35. Zeni, C.; Rossi, K.; Glielmo, A.; de Gironcoli, S. Compact atomic descriptors enable accurate predictions via linear models. J. Chem. Phys. 2021, 154, 224112. [CrossRef]

36. Artrith, N. Machine learning for the modeling of interfaces in energy storage and conversion materials. J. Phys. Energy 2019, 1, 032002. [CrossRef]

37. Schleder, G.R.; Padilha, A.C.M.; Acosta, C.M.; Costa, M.; Fazzio, A. From DFT to machine learning: Resent approaches to materials science-A review. J. Phys. Mater. 2019, 2, 032001. [CrossRef]

38. Massart, D.L.; Kaufman, L. The Interpretation of Analytical Chemical Data by the Use of Cluster Analysis; John Wiley and Sons: New York, NY, USA, 1983

39. Vandeginste, B.; Massart, D.; de Jong, S.; Buydens, L. Handbook of Chemometrics and Qualimetrics: Part B; Elsevier: Amsterdam, The Netherlands, 1998.

40. Hill, T.; Lewicki, P. STATISTICS: Methods and Applications; StatSoft, Inc.: Tulsa, OK, USA, 2007.

41. Clar, E.; Kemp, W.; Stewart, D. The significance of Kekulé structures for the stability of aromatic systems. Tetrahedron 1958, 3 , 325-333. [CrossRef]

42. Witek, H.A. Zhang-Zhang polynomials of multiple zigzag chains revisited: A connection with the John-Sachs theorem. Molecules 2021, 26, 2524. [CrossRef] [PubMed] 